\title{
Management of Weight Gain in Midlife Women: Hormone Therapy and Beyond
}

\author{
Ekta Kapoor, MBBS, FACP, ${ }^{1,2}$ Julia A. Files, MD, FACP, and Stephanie S. Faubion, MD ${ }^{1}$
}

\section{Case History}

A 52-YEAR-OLD woman presents to your office with concerns about weight gain. She experienced her last menstrual period at the age of 49 , and reports gaining 8 pounds since then. She is very distressed about her weight, particularly the weight gain in her midsection. She indicates that she has not changed anything about her diet or exercise in the past 3 years, yet she continues to gain weight. She also reports bothersome hot flashes and night sweats, which are interfering with her ability to function during the day. She wonders about using menopausal hormone therapy (MHT), but is concerned it will cause her to gain more weight. Her medical history is remarkable for impaired fasting glucose and dyslipidemia. She is on no medications. Her physical examination reveals a BMI of 27.6 and blood pressure of 136/88.

How do you advise her?

A. You explain that the extra weight will have little impact on her overall health.

B. You indicate that she could use MHT, but that it might result in additional weight gain, particularly around the midsection.

C. You advise more exercise as an effective means for taking off the extra weight.

D. You report that MHT would be effective for management of hot flashes and night sweats and does not contribute to weight gain.

E. You explain that menopause is the primary reason for her weight gain.

Weight gain is a common complaint among midlife women. ${ }^{1}$ In addition to increased total body fat, postmenopausal women very commonly experience body fat distribution changes with a tendency for central fat deposition (android pattern), versus the lower body (gynoid pattern) that is characteristic of premenopausal women. ${ }^{2}$ These changes in body composition and fat distribution are likely responsible, at least to some extent, for the increased risk of cardiovascular disease (CVD) in postmenopausal women. Several studies demonstrate a correlation between earlier age of menarche and increased risk of midlife obesity.

The majority of the current literature supports that weight gain at midlife is predominantly an aging effect. ${ }^{3}$
There is a natural tendency for weight gain in both sexes with aging. This is because of a decrease in lean body mass (LBM) and a decrease in physical activity (PA) level. These factors contribute to fall in both the resting and total energy expenditure, ${ }^{4,5}$ and unless there are compensatory changes in dietary habits and/or energy expenditure, there is a state of positive energy balance, which results in weight gain. In addition, perimenopausal women experience a variety of symptoms that may compromise their ability to adhere to a healthy lifestyle, including vasomotor symptoms, sleep disturbances, and mood disorders, thereby facilitating weight gain. ${ }^{6,7}$ In addition, the postmenopausal drop in estrogen has a significant effect on adipocyte metabolism, sex-specific remodeling of adipose depots, control of appetite and satiety, and energy expenditure. ${ }^{8}$ Postmenopausal women preferentially deposit excess body fat in the midsection, an effect that persists despite adjustment for aging, total body fat, and PA level, all of which are independently associated with increased abdominal fat. ${ }^{9}$

Surgical menopause may increase the risk of obesity compared with natural menopause. In the Study of Women's Health Across the Nation, the odds of developing severe obesity were increased five times in women who underwent surgical menopause compared with those who were premenopausal. ${ }^{10}$

Obesity has significant impact on the physical and emotional health of midlife women. Central obesity is associated with a variety of adverse metabolic complications including dysglycemia or frank type 2 diabetes, dyslipidemia, hypertension (these three constitute the metabolic syndrome), and ultimately increased risk of CVD., ${ }^{9,11}$ Obesity is also associated with increased risk for certain cancers (breast and uterine), and a higher risk of overall cancer-related mortality. ${ }^{12,13}$ Other disorders associated with weight gain include obstructive sleep apnea, gastroesophageal reflux disease, osteoarthritis, cholelithiasis, and asthma. Obese women are more likely to experience severe vasomotor symptoms and are also more likely to report them. ${ }^{14}$ Weight loss, in contrast, is associated with improvement in hot flashes. ${ }^{15}$ Weight gain in the climacteric years can also compromise a woman's emotional and psychosocial well-being, leading to partner relationship issues and sexual dysfunction. ${ }^{16-18}$

\footnotetext{
${ }^{1}$ Division of General Internal Medicine, Women's Health Clinic, Mayo Clinic, Rochester, Minnesota.

${ }^{2}$ Division of Endocrinology, Metabolism and Nutrition, Mayo Clinic, Rochester, Minnesota.

${ }^{3}$ Division of Women's Health-Internal Medicine, Mayo Clinic in Arizona, Scottsdale, Arizona.
} 
Weight management in midlife women is crucial for the reasons already stated. Providers caring for women need to be proactive in screening for obesity and offering counseling for weight management even if not specifically sought by the patient. An ideal weight management program follows a multidisciplinary approach and involves multiple experts, including medical providers, behavioral psychologists, dietitians, and lifestyle coaches. In addition to standard advice regarding a hypocaloric diet (about $500-750 \mathrm{kcal}$ deficit/ day), adequate intake of whole grains, fruits and vegetables, and regular exercise (150-175 minutes/week), it is very important to recognize and manage any potential barriers to compliance with healthy lifestyle measures. These include vasomotor symptoms, sleep disturbances, mood problems, degenerative joint disease, and poor social support. Providers caring for midlife women should have a good understanding of menopausal symptoms and their management. As long as the patient is adherent to a hypocaloric diet, the macronutrient composition of the diet does not have a bearing on the weight outcome. ${ }^{19}$

Questions about the relationship between MHT and weight gain often come up in clinical practice. In the absence of contraindications, MHT is recommended for recently menopausal women with moderate to severe vasomotor symptoms. Although MHT does not affect weight, it does result in an improved body composition (more LBM and less visceral fat), lowered insulin resistance, and an improved lipid profile. ${ }^{20}$ However, MHT use cannot be recommended for weight management or CVD prevention. Women who use MHT for other reasons can, nevertheless, be counseled about its beneficial effects on body composition. Also, MHT use in younger postmenopausal women who are within 10 years of their final menstrual period does not appear to increase the risk of CVD, and may even be protective. ${ }^{21}$

Weight management is a lifelong journey that is primarily focused on changing behaviors. Patients require psychological support, motivation, and education regarding selfmonitoring, problem solving, strategizing, and reinforcement, and the expertise of a behavioral psychologist specializing in obesity may be of benefit to the patient, if available.

Although initial weight loss may be relatively easy with intensive lifestyle changes, maintaining weight loss may be more difficult. This is mostly because of the fall in the metabolic rate with dieting-induced weight loss, and compensatory hormonal changes that adversely affect appetite and satiety. Patients, therefore, are required to intensify their efforts at dietary change and PA to maintain weight loss. Behaviors that predict weight regain include poor social support, psychiatric comorbidities, emotional eating, and decreased PA.

In summary, midlife women experience a unique set of problems that increase the odds of weight gain and unfavorable body fat distribution beyond what is brought on by aging alone. Weight management in midlife women requires careful assessment and management of comorbidities that facilitate weight gain. MHT used for management of vasomotor symptoms may result in favorable weight distribution changes, but will not result in weight loss.

\section{Answer: D}

Answer A is incorrect as she has metabolic syndrome (overweight, elevated blood pressure, dyslipidemia, and im- paired fasting glucose), which increases her risk for CVD, the leading cause of death in postmenopausal women. Answer B is incorrect as MHT does not cause weight gain, and may result in more favorable fat distribution (away from the midsection). Answer $\mathrm{C}$ is incorrect as exercise alone has not been shown to result in significant weight loss, although it is beneficial for weight maintenance. Answer E is incorrect as weight gain in midlife women is primarily an effect of aging rather than of menopause.

\section{References}

1. Flegal KM, Carroll MD, Ogden CL, Curtin LR. Prevalence and trends in obesity among US adults, 1999-2008. JAMA 2010;303:235-241.

2. Toth MJ, Tchernof A, Sites CK, Poehlman ET. Menopauserelated changes in body fat distribution. Ann N Y Acad Sci 2000;904:502-506.

3. Macdonald HM, New SA, Campbell MK, Reid DM. Longitudinal changes in weight in perimenopausal and early postmenopausal women: Effects of dietary energy intake, energy expenditure, dietary calcium intake and hormone replacement therapy. Int J Obes Relat Metab Disord 2003; 27:669-676.

4. Douchi T, Yamamoto S, Yoshimitsu N, Andoh T, Matsuo $\mathrm{T}$, Nagata $\mathrm{Y}$. Relative contribution of aging and menopause to changes in lean and fat mass in segmental regions. Maturitas 2002;42:301-306.

5. Poehlman ET, Toth MJ, Bunyard LB, et al. Physiological predictors of increasing total and central adiposity in aging men and women. Arch Intern Med 1995;155:24432448.

6. Patel SR, Malhotra A, White DP, Gottlieb DJ, Hu FB. Association between reduced sleep and weight gain in women. Am J Epidemiol 2006;164:947-954.

7. Bromberger JT, Matthews KA, Schott LL, et al. Depressive symptoms during the menopausal transition: The Study of Women's Health Across the Nation (SWAN). J Affect Disord 2007;103:267-272.

8. Lizcano F, Guzman G. Estrogen deficiency and the origin of obesity during menopause. Biomed Res Int 2014;2014: 757461.

9. Folsom AR, Kushi LH, Anderson KE, et al. Associations of general and abdominal obesity with multiple health outcomes in older women: The Iowa Women's Health Study. Arch Intern Med 2000;160:2117-2128.

10. Sutton-Tyrrell K, Zhao X, Santoro N, et al. Reproductive hormones and obesity: 9 years of observation from the Study of Women's Health Across the Nation. Am J Epidemiol 2010;171:1203-1213.

11. Manson JE, Willett WC, Stampfer MJ, et al. Body weight and mortality among women. N Engl J Med 1995;333: 677-685.

12. Lauby-Secretan B, Scoccianti C, Loomis D, et al. Body fatness and cancer-Viewpoint of the IARC Working Group. N Engl J Med 2016;375:794-798.

13. Calle EE, Rodriguez C, Walker-Thurmond K, Thun MJ. Overweight, obesity, and mortality from cancer in a prospectively studied cohort of U.S. adults. N Engl J Med 2003;348:1625-1638.

14. Thurston RC, Sowers MR, Sternfeld B, et al. Gains in body fat and vasomotor symptom reporting over the menopausal transition: The study of women's health across the nation. Am J Epidemiol 2009;170:766-774. 
15. Kroenke $\mathrm{CH}$, Caan BJ, Stefanick ML, et al. Effects of a dietary intervention and weight change on vasomotor symptoms in the Women's Health Initiative. Menopause 2012;19:980-988.

16. Faubion SS, Rullo JE. Sexual dysfunction in women: A practical approach. Am Fam Physician 2015;92:281-288.

17. Nackers LM, Appelhans BM, Segawa E, Janssen I, Dugan SA, Kravitz HM. Associations between body mass index and sexual functioning in midlife women: The study of women's health across the nation. Menopause 2015;22: 1175-1181.

18. Simoncig Netjasov A, Tancic-Gajic M, Ivovic M, Marina L, Arizanovic Z, Vujovic S. Influence of obesity and hormone disturbances on sexuality of women in the menopause. Gynecol Endocrinol 2016;32:762-766.

19. Sacks FM, Bray GA, Carey VJ, et al. Comparison of weight-loss diets with different compositions of fat, protein, and carbohydrates. N Engl J Med 2009;360:859-873.

20. Salpeter SR, Walsh JM, Ormiston TM, Greyber E, Buckley NS, Salpeter EE. Meta-analysis: Effect of hormone- replacement therapy on components of the metabolic syndrome in postmenopausal women. Diabetes Obes Metab 2006;8:538-554.

21. Manson JE, Chlebowski RT, Stefanick ML, et al. Menopausal hormone therapy and health outcomes during the intervention and extended poststopping phases of the Women's Health Initiative randomized trials. JAMA 2013; 310:1353-1368.

Address correspondence to: Ekta Kapoor, MBBS, FACP Women's Health Clinic Division of General Internal Medicine Division of Endocrinology, Metabolism and Nutrition Mayo Clinic Rochester, MN 55905

E-mail: kapoor.ekta@mayo.edu 